\title{
Substrate-Integrated Waveguide Band Pass Filters with Frequency-Dependent Coupling Elements
}

\author{
Mehdi Salehi, ${ }^{1,2}$ Jens Bornemann, ${ }^{1}$ Esfandiar Mehrshahi ${ }^{2}$ \\ ${ }^{1}$ Department of Electrical and Computer Engineering, University of Victoria, Victoria, BC, Canada \\ ${ }^{2}$ Department of Electrical and Computer Engineering, Shahid Beheshti University, G.C., Tehran, Iran
}

Received 20 December 2012; accepted 19 March 2013

\begin{abstract}
An effective technique to improve the stop-band frequency response of directcoupled resonators in substrate-integrated waveguide (SIW) technology is introduced. Regular inductive-iris filters in SIW technology are supplemented with H-plane frequency-dependent inverters which not only create transmission zeros but also serve as the proper impedance inverter. A synthesis technique is introduced to prescribe transmission zeros at finite frequencies on either side of the pass band, symmetrically or asymmetrically. Two different topologies of frequency-dependent inverters for X-band SIW band pass filters demonstrate that attenuation poles can be created on both side of the passband and significantly improve the filters' stop-band performences. Measurements confirm the validity of the presented design approach. (c) 2013 Wiley Periodicals, Inc. Int J RF and Microwave CAE 24:237-242, 2014.
\end{abstract}

Keywords: substrate-integrated waveguide; band pass filter; frequency-dependent inverter; transmission zero

\section{I.INTRODUCTION}

In modern microwave and wireless communication systems, the demand for band pass filters with high performance parameters such as stringent frequency selectivity, low insertion loss, wide stop-band, and especially potential integration into RF circuits is ever increasing. Substrate-integrated waveguide (SIW) is a low-cost realization of traditional waveguide circuits that inherits the merits from both the traditional microstrip technology for easy planar circuit integration and waveguide for low radiation loss and high quality factor. Hence, this technology provides high promise for future microwave planar circuits, components and subsystems [1-3].

Realizing SIW band pass filters with sharp cut-off skirts, the standard solution consists of generating transmission zeros on each side of the passband. It is traditionally accomplished by utilizing cross coupling or dual/ multimode cavities [4-6]. However, they need additional coupling between nonadjacent resonators, which often requires changes to the inline topology of resonators and complicates the general filter topology.

Correspondence to: M. Salehi; e-mail: msalehi@uvic.ca. DOI: $10.1002 /$ mmce. 20754

Published online 12 July 2013 in Wiley Online Library (wileyonlinelibrary.com).
Therefore, a high-performance mechanism is presented in this article that generates finite transmission zeros in direct-coupled resonator band pass filters without any additional coupling between nonadjacent resonators. Selected coupling sections, which in SIW inline filter configurations are realized by inductive irises, are replaced by structures that strongly depend on frequency in order to generate transmission zeros and, at the same time, provide proper inverter values for the coupling of resonators in the filter [7,8]. A frequency-dependent inverter can be realized as an H-plane stub or a shorted T-junction. Such coupling elements simultaneously provide a transmission zero and the correct inverter value for the filter design. Conversely, $\mathrm{H}$-plane stubs can be added to the filter for the sole purpose of providing transmission zeros. The dimensions of the shorted stubs offer the freedom to introduce attenuation poles at any prescribed frequencies, either symmetrically or asymmetrically with respect to the pass band. A design procedure for pre-synthesized frequency-dependent coupling elements is presented. Then, to demonstrate the advantages of the method, two fourthorder SIW filters with bandwidth of $500 \mathrm{MHz}$ at $11 \mathrm{GHz}$ are designed on $0.508 \mathrm{~mm}$ RT/Duroid 5870 substrate and verified using the High Frequency Structure Simulator (HFSS), Computer Simulation Technology (CST), and measurements.

(C) 2013 Wiley Periodicals, Inc. 


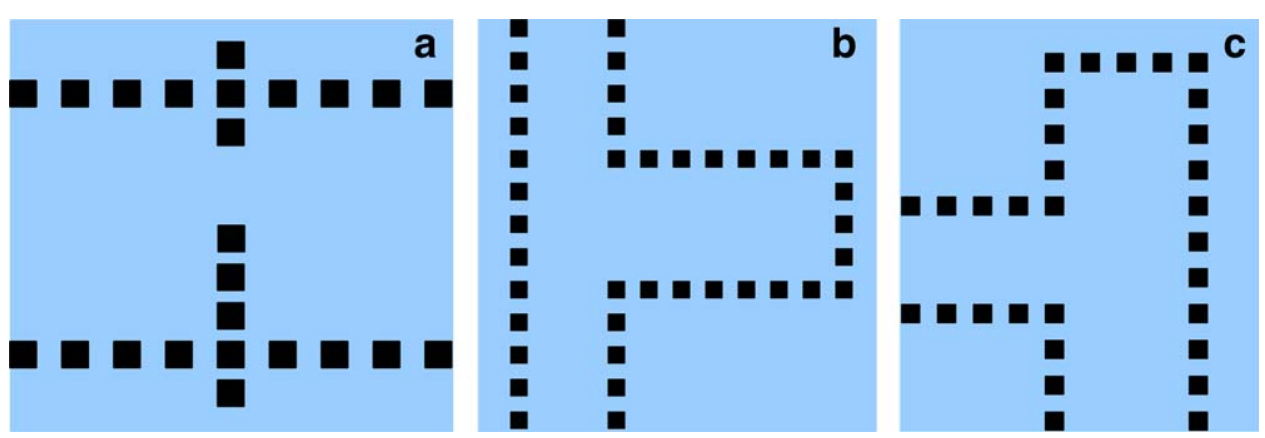

Figure 1 SIW impedance inverters with square via holes; (a) asymmetric iris, (b) H-plane stub, (c) shorted T-junction.

\section{II.DESIGN METHODOLOGY}

\section{A.Inverter Model}

The synthesis procedures for the design of directcoupled resonator SIW band pass filters with frequencydependent coupling elements are presented. Impedance inverters, which can be realized as standard irises, frequency-dependent H-plane stubs, and frequencydependent T-junctions, as shown in Figure 1, are employed as coupling elements to produce transmission zeros.

The design of a regular waveguide filter, which consists of a number of in-line resonators, commences with the calculation and realization of its inverter values, for example, Ref. 9. This procedure takes place at center frequency, where the equivalent-circuit model shown in Figure 2 is valid for all three inverters of Figure 1, and the reference planes are determined by the $\pm 90^{\circ}$ phase shift required for inverter operation.

Once the theoretical inverter values $K$ are obtained from filter theory ([9], p. 182), the shunt reactance $X_{\mathrm{p}}$, the two series reactances $X_{\mathrm{s} 1}, X_{\mathrm{s} 2}$ and associated phases $\varphi_{1}$ and $\varphi_{2}$ (Fig. 2) of the actual inverters of Figure 1 must be designed to match the theoretical $K$ values from filter synthesis at center frequency. This is done according to the following procedure.

First, the fundamental-mode scattering parameters of the inverters in Figure 1 are calculated using the mode matching technique ([9], Chapter 2.1, Chapter 3.5.4 and [10]) for fixed parameters of square via length $L_{\text {square }}$ and via center-to-center spacing $s$. These scattering parameters are then converted to inverter values according to equations listed below

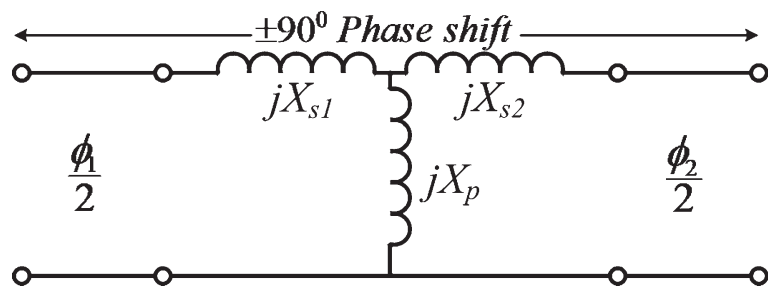

Figure 2 Equivalent circuit model of the structures in Figure 1.

$$
\begin{aligned}
& j X_{s 1}=\frac{\left[1+S_{11}\right]\left[1-S_{22}\right]+S_{21}^{2}-2 S_{21}}{\left[1-S_{11}\right]\left[1-S_{22}\right]-S_{21}^{2}} \\
& j X_{\mathrm{s} 2}=\frac{\left[1-S_{11}\right]\left[1+S_{22}\right]+S_{21}^{2}-2 S_{21}}{\left[1-S_{11}\right]\left[1-S_{22}\right]-S_{21}^{2}} \\
& j X_{\mathrm{p}}=\frac{2 S_{21}}{\left[1-S_{11}\right]\left[1-S_{22}\right]-S_{21}^{2}} \\
& {\left[\begin{array}{c}
\varphi_{1} \\
\varphi_{2}
\end{array}\right]=-\arctan \left\{\frac{X_{\mathrm{s} 1}+X_{\mathrm{s} 2}+2 X_{\mathrm{p}}}{1-X_{\mathrm{s} 1} X_{\mathrm{s} 2}-X_{\mathrm{p}}\left(X_{\mathrm{s} 1}+X_{\mathrm{s} 2}\right)}\right\} } \\
&=\arctan \left\{\frac{X_{\mathrm{s} 1}-X_{\mathrm{s} 2}}{1+X_{\mathrm{s} 1} X_{\mathrm{s} 2}+X_{\mathrm{p}}\left(X_{\mathrm{s} 1}+X_{\mathrm{s} 2}\right)}\right\} \\
& K=\sqrt{\frac{1+\Gamma \exp \left(-j \varphi_{1}\right)}{1-\Gamma \exp \left(-j \varphi_{1}\right)}}
\end{aligned}
$$

where

$$
\Gamma=\frac{\left[j X_{\mathrm{s} 1}+j X_{\mathrm{p}}-1\right]\left[j X_{\mathrm{s} 2}+j X_{\mathrm{p}}+1\right]+X_{\mathrm{p}}^{2}}{\left[j X_{\mathrm{s} 1}+j X_{\mathrm{p}}+1\right]\left[j X_{\mathrm{s} 2}+j X_{\mathrm{p}}+1\right]+X_{\mathrm{p}}^{2}}
$$

Note that for inverters of Figures $1 \mathrm{a}$ and $1 \mathrm{~b}$, the equivalent-circuit parameters will be symmetric, that is, $X_{\mathrm{s} 1}=X_{\mathrm{s} 2}$ and $\varphi_{1}=\varphi_{2}$. Only the inverter of Figure $1 \mathrm{c}$ produces an asymmetric circuit.

If the computed inverter value does not agree with the theoretical one from filter synthesis, then the parameters of the inverter values in Figure 1 are varied until the two inverter values match. The parameters to be varied are: the aperture width for the inverter in Figure 1a, the stub width or stub length for the inverter in Figure $1 b$, and the length of the shorted stub in Figure 1c.

To highlight the different frequency dependencies of the inverters, Figure 3 compares the scattering parameters of the three inverters in Figure 1 that have been designed for the same center frequency. In the frequency band around 11 $\mathrm{GHz}$, all three inverters show nearly identical behavior. However, away from the center frequency, their behavior is very different. The asymmetric iris (solid lines) shows the smallest variation. Therefore, the coupling values of inductive irises 


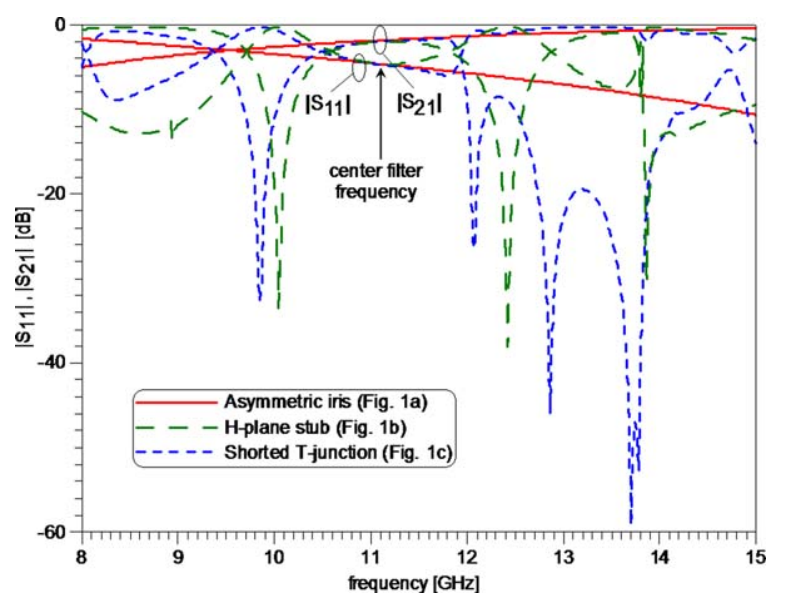

Figure 3 Scattering parameters of asymmetric iris (Fig. 1a), H-plane stub (Fig. 1b), and shorted T-junction (Fig. 1c).

are usually considered nearly constant over a wider frequency range. The H-plane stub (dashed lines) acts as an inverter in the vicinity of the filter pass band but produces two transmission zeros (TZs) immediately to the left and the right of the pass band. These TZs are created at the resonant frequencies of the stub. Similarly, the shorted T-junction (dashed lines) acts as an inverter in the pass band but produces different and more TZs than the H-plane stub. Such TZs are used to improve the out-of-band frequency response of the filter. Their frequency positions can be controlled by varying the geometrical parameters of the stub or shorted T-junction while maintaining the proper inverter values at center frequency.

\section{B.Filter Design Procedure}

The first step in the filter design procedure is to design a filter in SIW technology with all inverters realized by inductive irises as coupling sections.

A search algorithm varies the dimension of the coupling section until the normalized prototype inverter values $K$ in (3) match the prototype inverters $K_{\text {prototype }}$ obtained from standard Chebychev filter synthesis [11]. This process is repeated for all coupling sections, either irises or stubs, of the filter, and the distances between adjacent inverters are adjusted according to Ref. 11 .

The filter is initially synthesized with all inductive irises, and the equivalent waveguide lengths and widths are converted to the respective SIW parameters [12]. Then a coupling element, which will be converted to a frequency-dependent inverter, is selected. This coupling element can be any iris in the filter. However, for faster design (optimization), the outermost elements are usually easier to match to the rest of the filter. In order to prescribe one or two transmission zeros at frequencies $f_{\mathrm{z} 1}$ and $f_{\mathrm{z} 2}$ for an inverter at $f_{0}$, the inverter dimensions (referred to as dim in Eqn. 5) are optimized by minimizing the function

$$
\begin{aligned}
F_{\text {min }}=\left[K_{\text {prototype }}\right. & \left.-K_{\text {dispersive }}\left(f_{0}, \operatorname{dim}\right)\right]^{2} \\
& +w_{n 1}\left|S_{21}\left(f=f_{z 1}\right)\right|^{2}+w_{n 2}\left|S_{21}\left(f=f_{z 2}\right)\right|^{2}
\end{aligned}
$$

where $w_{\mathrm{n}}$ are individual weights for the transmission zeros at $f_{\mathrm{zn}}$.

Frequency-dependent H-plane stubs can replace any iris in the filter as inverter [13] or, in a special case, they can be used for the sole purpose of providing more transmission zeros. When a frequency-dependent element $(\mathrm{H}-$ plane stub) is added at one end of a filter without simultaneously acting as an inverter but producing $N$ TZs at frequencies $f_{\mathrm{zn}}$, the function to be minimized is modified as

$$
F_{\min }=\sum_{i}\left[1-\left|S_{21}\left(f_{i}\right)\right|\right]^{2}+\sum_{n}^{N} w_{n}\left|S_{21}\left(f=f_{z n}\right)\right|^{2}
$$

where, $f_{\mathrm{i}}$ are the passband frequency samples.

A final fine optimization of the entire filter structure is required to fine tune the return loss while maintaining the transmission zeros. As circular via holes are more commonly fabricated than square ones, every square via in the filter may be replaced by a circular via hole using the relation of [14].

$$
d_{\text {circle }}=\frac{2 L_{\text {square }}}{1+1 / \sqrt{2}}
$$

Good initial values and the proper cost function are critical factors in optimization convergence. The frequency points are selected in such a way that scattering parameters are evaluated only at band edges as well as at the transmission and reflection zeros [15]. This approach is useful for full-wave optimization at very few frequency points, which increases convergence speed. However, if the initial values are off or the transmission zeros are very close to the pass band, then the cost function may not provide a good return loss in the filter pass band.

\section{III.RESULTS AND DISCUSSION}

Following the synthesis procedure, two SIW X-band filters involving frequency-dependent inverters have been designed and prototyped on RT/Duroid 5870 with a thickness of $0.508 \mathrm{~mm}$, permittivity of 2.33 , conductor thickness of $17.5 \mu \mathrm{m}$ and loss tangent of 0.0012 . The circular via diameter and center-to-center via spacing are chosen as 1 and $1.5 \mathrm{~mm}$, respectively.

\section{A.Using H-Plane Stub for Added Transmission Zeros and T-Junction as Inverter}

To present the individual TZs created by frequencydependent inverters, we first proceed with the design of a fourth order in-line SIW filter with all inductive irises according to Figure 1a. The filter is designed at $11 \mathrm{GHz}$ with a band width of $500 \mathrm{MHz}$. The transmission performance of this filter is depicted as solid lines in Figure 


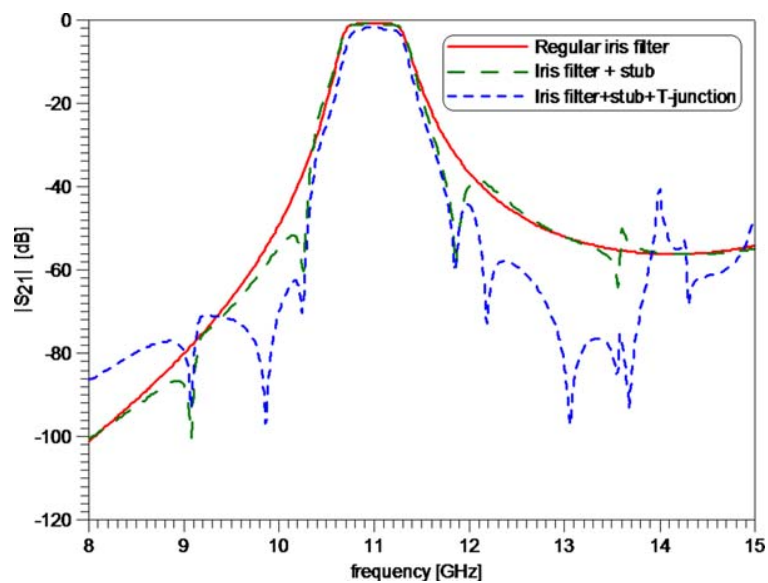

Figure 4 Transmission parameters for design of in-line forthorder SIW filter (solid line), adding H-plane stub before the first iris for producing transmission zeros (dashed line), and supplementing the last iris window by adding a shorted T-junction (dotted line).

4. In the second step, an H-plane stub is optimized (by changing its width and length) to produce three determined TZs at 9.2, 10.25, and $11.85 \mathrm{GHz}$. This stub is added to the left side of the filter (c.f., Fig. 5a). As shown by the dashed line in Figure 4, the three transmission zeros are generated and added to the filter performance.

Finally, a shorted T-junction, which acts as a proper inverter and produces two additional TZs at 9.88 and $10.22 \mathrm{GHz}$, is optimized and imported at the right side of the circuit (Fig. 5a) and replaces the last iris inverter. As illustrated by the dotted line in Figure 4, the two TZs are added to the previous frequency response. Note that the return loss is not shown in Figure 4 since the addition of frequency-dependent inverters also requires that the adjacent resonators and irises be re-optimized to account for the different loads presented to the filter. In a first optimization step, only the inductive irises next to the frequency-dependent inverters and the lengths between them are varied. Then, the dimensions of the filter are optimized for the best return loss. This produces an appropriate tuning of the resonators next to the frequencydependent stubs. A final fine optimization includes the remaining parameters of the filter but excludes the stub dimensions to maintain the position of the transmission zeros [15]. The other TZs shown in Figure 4 at higher frequencies are generated due to higher-order mode resonances. Although they are not controllable while keeping the previous standard TZs, they can effectively improve the stop band performance of the filter.

Figure 5a shows the layout and dimensions of a fourth-order SIW band pass filter at $11 \mathrm{GHz}$ with a band width of $500 \mathrm{MHz}$ which generates six TZs between 9 and $13.5 \mathrm{GHz}$. Note that all-dielectric waveguide ports of equivalent width $13.9 \mathrm{~mm}$ are used since they avoid the influence of transitions to affect the filter performance [16]. This width of $13.9 \mathrm{~mm}$ is the width of an all-dielectric waveguide that, when connected to the center-to-center SIW width of $14.6 \mathrm{~mm}$ (Fig. 5a) produces reflection that is in the order of $10 \mathrm{~dB}$ lower than that created by the filter [17]. The all-dielectric waveguide width is related to the SIW width by a closed-form expression in Ref. 12. In the measurements of the prototype shown in the inset of Figure 5b, the SIW-to-microstrip transitions, which are designed according to Ref. 18, are deembedded by using a set of TRL calibration standards.

Results using HFSS and CST are shown in Figure 5b and are in very good agreement. In this design, only the shorted T-junction at the lower right side of the circuit is used as a frequency-dependent inverter which generates the transmission zeros at 9.88 and $12.22 \mathrm{GHz}$. The three attenuation poles between 9 and $12 \mathrm{GHz}$ are generated by the additional H-plane stub which does not act as an inverter. The shorted stubs produce extra attenuation poles at higher frequencies and hence, as shown in Figure 5b, effectively improve the stop band of the filter.

The measurements confirm the predicted results by HFSS and CST in principle. First of all, a slight shift
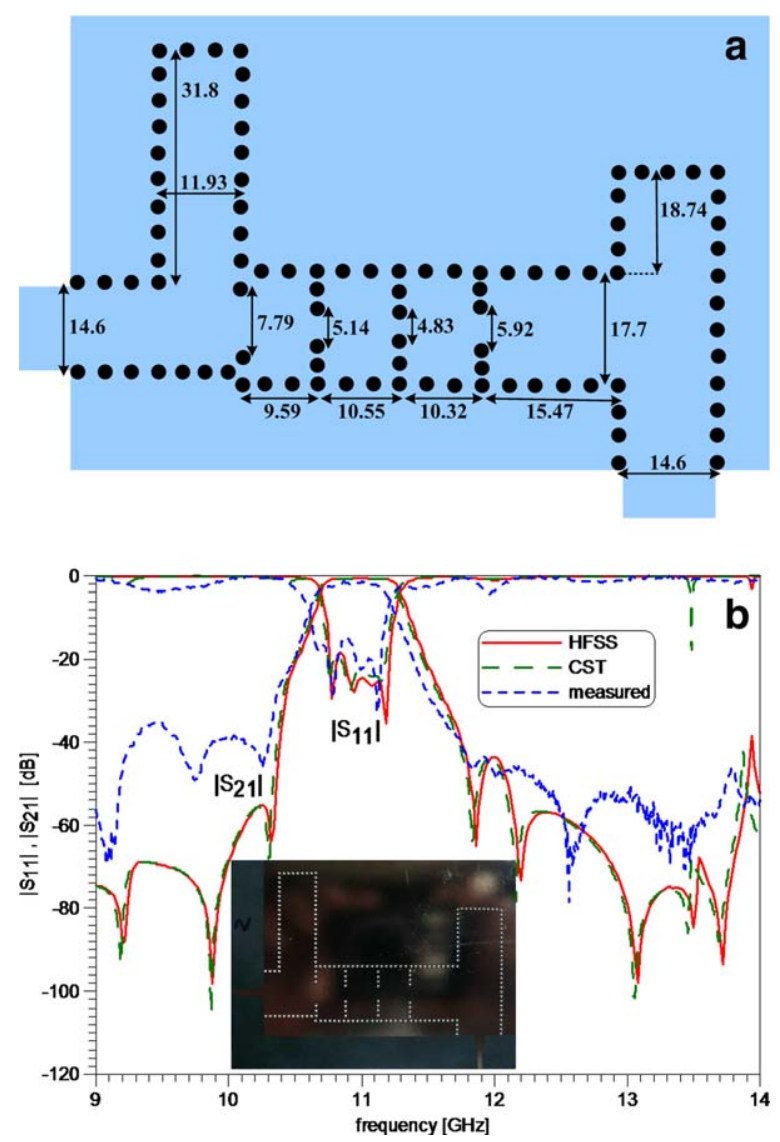

Figure 5 Layout and frequency response of an X-band SIW filter with shorted T-junction inverter and H-plane stub. (a) Layout with dimensions in $\mathrm{mm}$ (actual number of via holes according to the inset in Fig. 5b); (b) frequency response including comparison between HFSS, CST, and measurements. 

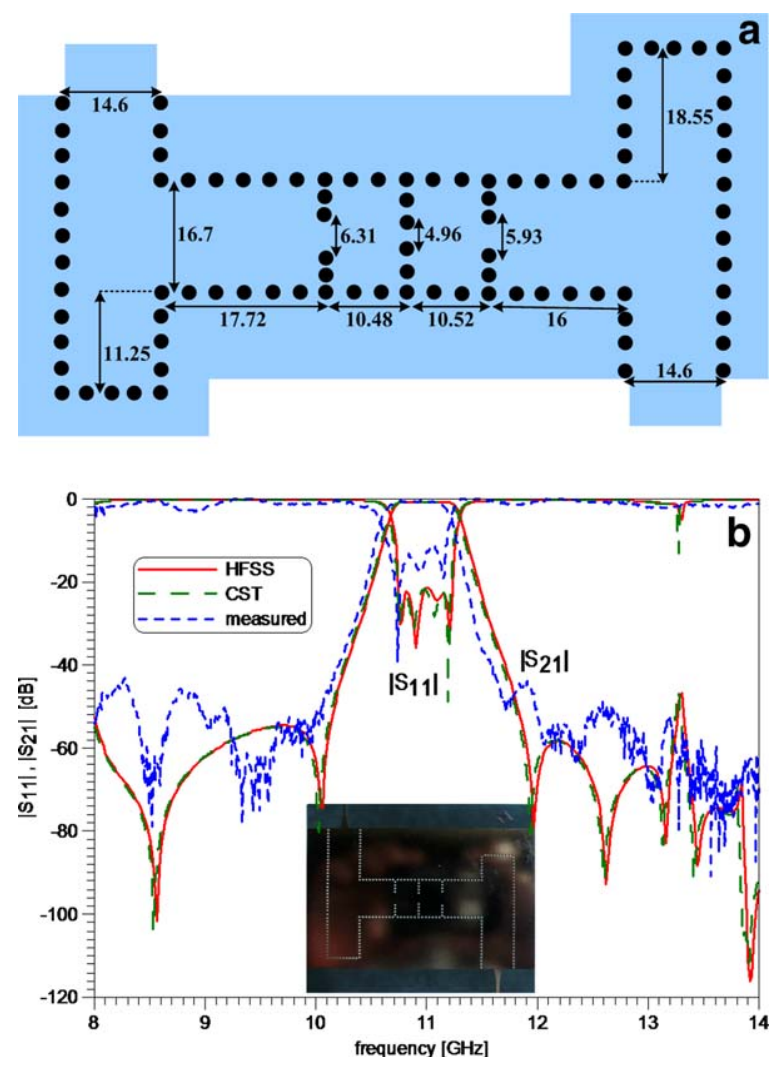

Figure 6 Layout and frequency response of an X-band SIW filter with two shorted T-junction inverters. (a) Layout with dimensions in $\mathrm{mm}$ (actual number of via holes according to the inset in Fig. 6b); (b) frequency response including comparison between HFSS, CST, and measurements.

toward lower frequencies is observed. This is due to the fact that the via holes are slightly smaller than the specifications. Therefore, the resonators are wider and longer and thus move the filter response downwards. This also explains the increased return loss in the pass band $(15 \mathrm{~dB}$ measured compared to $18 \mathrm{~dB}$ in HFSS). The pass band insertion loss is measured as $1.5 \mathrm{~dB}$ while the predicted value is $0.75 \mathrm{~dB}$. The measured upper stop band response is reasonable considering the fact that values are in the order of $50 \mathrm{~dB}$. In the lower stop band, the transmission zeros are well represented in the experimental results. However, the level of attenuation is not reproduced in the measurements. As there is also a $3.6 \mathrm{~dB}$ dip in the reflection coefficient values, it is believed that the differences are caused by the calibration standards and the deembedding process. Note that radiation from the filter or the microstrip transitions, which are connected to SMA end launchers, has not been observed.

\section{B.Using Two T-Junctions as Inverters}

In this design, the outermost irises of the initial design (solid line in Fig. 4) are replaced by shorted T-junction inverters to produce a fourth-order SIW band pass filter at
$11 \mathrm{GHz}$ with the same bandwidth and one symmetric and one asymmetric TZs at each side of the pass band. Two shorted T-junctions, which serve as proper inverter values, are designed and optimized to produce symmetric transmission zeros at 10 and $12 \mathrm{GHz}$, and asymmetric transmission zeros at 8.6 and $12.6 \mathrm{GHz}$. The layout of the filter is depicted in Figure 6a.

As it shows, the first and last coupling sections consist of two shorted T-junction inverters that each provide two TZs. The width of the stubs is fixed to that of the ports. Therefore, by changing the length of each shorting stub, TZs can be realized in the lower or upper stop bands of the filter. As the length of the stubs increase, the positions of the TZs decrease. Since the shorted sections are longer than a wavelength, they produce a number of TZs. However, only one of them can be controlled to fall in the frequency range between 9.95 and $12.05 \mathrm{GHz}$. The other ones appear at higher-order resonances of the stubs. By controlling one $\mathrm{TZ}$ of each $\mathrm{T}$-junction inverter to fall at frequencies symmetric to the passband, as shown in Figure $6 \mathrm{~b}$, symmetric skirts with sharp cutoffs characteristic can be achieved. Note that such inverter structures have the flexibility to be used in both broadband and narrowband filters.

Similar to the filter in Figure 5, the measurements in Figure $6 \mathrm{~b}$ verify the design process in principle, and arguments about the prototype and experimental setup hold accordingly. The comparison between measurements and simulation are as follows: The measured in-band return loss is $12 \mathrm{~dB}$ compared to $21 \mathrm{~dB}$ in the simulations. The average measured insertion loss of $0.8 \mathrm{~dB}$ agrees well with predictions by HFSS and CST.

\section{IV.CONCLUSION}

A design procedure for the synthesis of frequencydependent coupling elements in SIW band pass filters with direct-coupled resonators is presented. Frequencydependent inverters can simultaneously provide attenuation poles and proper inverter values for SIW filter design. A mixture of irises and stubs are used in the same design as coupling elements, but only the SIW stubs provide attenuation poles on either side of the passband while maintaining the coupling required in the filter. Two fourth-order SIW band pass filters at $11 \mathrm{GHz}$ and bandwidth of $500 \mathrm{MHz}$ are prototyped and illustrate the effectiveness of the technique for sharp skirt SIW filters with wide stop-bands. The designs are verified by commercially available field solvers and experimental results. Moreover, the proposed approach can potentially be used in microwave and millimeter-wave filter circuits using technologies other than SIW.

\section{ACKNOWLEDGMENTS}

The authors like to thank the Iran Telecommunication Research Center (ITRC) and the Natural Science and Engineering Research Council (NSERC) of Canada for supporting this research. 


\section{REFERENCES}

1. K.K. Samanta, D. Stephens, and I.D. Robertson, Design and performance of a 60-ghz multi-chip module receiver employing substrate integrated waveguides, IET Microw Antennas Propag 1 (2007), 961-967.

2. M. Salehi and E. Mehrshahi, Spurious-response suppression of substrate integrated waveguide filters using multishape resonators and slotted plane structures, Int J RF Microw CAE 21 (2011), 650-657.

3. V. Sekar, M. Armendariz, and K. Entesari, A 1.2-1.6-ghz substrate-integrated-waveguide rf mems tunable filter, IEEE Trans Microwave Theory Tech 59 (2011), 866-876.

4. Q.F. Wei, Z.F. Li, L. Li, W.J. Zhang, and J.F. Mao, Threepole cross-coupled substrate-integrated waveguide bandpass filters based on pcb process and multilayer ltcc technology, Microw Opt Technol Lett 51 (2009), 71-73.

5. Y. Dong, W. Hong, H. Tang, J. Chen, and K. Wu, Planar realization of a q-band triple-mode filter using high-order resonances, Microw Opt Technol Lett 51 (2009), 600-603.

6. D. Zelenchuk and V. Fusco, Low insertion loss substrate integrated waveguide quasi-elliptic filters for v-band wireless personal area network applications, IET Microw Antennas Propag 5 (2011), 921-927.

7. S. Amari and J. Bornemann, Using frequency-dependent coupling to generate finite attenuation poles in direct-coupled resonator bandpass filters, IEEE Microw Guided Wave Lett 9 (1999), 404-406.

8. S. Amari, J. Bornemann, W. Menzel, and F. Alessandri, Diplexer design using pre-synthesized waveguide filters with strongly dispersive inverters, IEEE MTT-S Int Microwave Symp Dig, vol. 3, Phoenix, AZ, 2001, 1627-1630.
9. J. Uher, J. Bornemann, and U. Rosenberg, Waveguide components for antenna feed systems. Theory and CAD, Artech House Inc., Norwood, MA, 1993.

10. J. Bornemann, F. Taringou, and Z. Kordiboroujeni, A modematching approach for the analysis and design of substrateintegrated waveguide components, Frequenz J RF/Microw Eng Photon Commun 65 (2011), 287-292.

11. G.L. Matthaei, L. Young, and E.M.T. Jones, Microwave filters, impedance-matching networks, and coupling structures, Artech House, Dedham, MA, 1980.

12. M. Salehi and E. Mehrshahi, A closed-form formula for dispersion characteristics of fundamental SIW mode, IEEE Microw Wireless Compon Lett 21 (2011), 4-6.

13. J. Bornemann and J. Uher, H-plane waveguide filters with Eplane dispersive inverters for high-power application, Proc. ANTEM/URSI, Ottawa, Canada, July 2004, 197-200.

14. Z. Kordiboroujeni, J. Bornemann, and T. Sieverding, Modematching design of substrate-integrated waveguide couplers, Proc. Asia-Pacific Int. Symp. Electromagnetic Compatibility, Singapore, May 2012, 701-704.

15. H.L. Thal Jr, Design of microwave filters with arbitrary responses, Int J RF Microw CAE 7 (1998), 208-221.

16. V.A. Labay and J. Bornemann, E-Plane directional couplers in substrate-integrated waveguide technology, Proc. AsiaPacific Microwave Conf., Hong Kong, December 2008, 1-4.

17. F. Taringou and J. Bornemann, Return-loss investigation of the equivalent width of substrate-integrated waveguide circuits, Proc. IEEE MTT-S Int. microwave workshop series millimeter wave integration technologies, Barcelona, Spain, September 2011, 703-706.

18. D. Deslandes, Design equations for tapered microstrip-tosubstrate integrated waveguide transitions, IEEE MTT-S Int. Microwave Symp Dig, Anaheim, CA, May 2010, 704-707.

\section{BIOGRAPHIES}

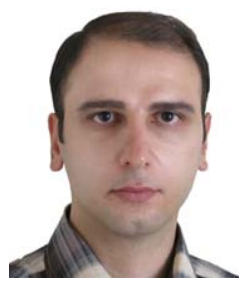

Mehdi Salehi was born in Isfahan, Iran, in 1979. He received the B.Sc. and M.Sc. degrees both in electrical engineering from Isfahan University of Technology (IUT), Isfahan, Iran, in 2001 and 2006, respectively. He has been appointed as research assistant at the University of Victoria, BC, Canada since 2011 to work on the design, analysis and synthesis of substrate integrated waveguide (SIW) structures. He received the Ph.D. in Electrical Engineering from Shahid Beheshti University (SBU), Tehran, Iran in 2013. He is currently working as a research associate at the University of Victoria. His current research interests include computer-aided design of RF and microwave structures, numerical methods in electromagnetics and advanced microwave and millimeter-wave circuits and components.

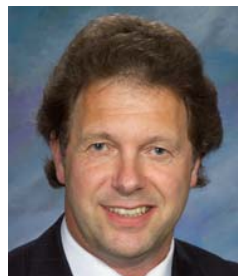

Jens Bornemann received the Dipl.-Ing. and the Dr.-Ing. degrees, both in electrical engineering, from the University of Bremen, Germany, in 1980 and 1984, respectively. From 1984 to 1985 , he was a consulting engineer. In 1985, he joined the University of Bremen, Germany, as an Assistant Professor. Since April 1988, he has been with the Department of Electrical and Computer Engineering, University of Victoria, Victoria, B.C., Canada, where he became a Professor in 1992. His research activities include RF/wireless/microwave/millimeter-wave components and systems design, and field-theory-based modeling of integrated circuits, feed networks, ultra-wideband technology and antennas. Dr. Bornemann is a Registered Professional Engineer in the Province of British Columbia, Canada. He is a Fellow IEEE, a Fellow of the Canadian Academy of Engineering (CAE) and serves on the editorial advisory board of the International Journal of Numerical Modelling.

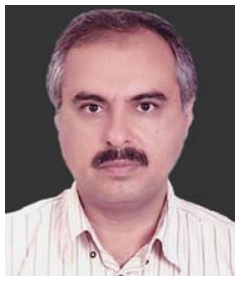

Esfandiar Mehrshahi was born in Tehran, Iran, in 1963. He received the B.Sc. degree from the Iran University of Science and Technology, Tehran, Iran, in 1987, and the M.Sc. and Ph.D. degrees from the Sharif University of Technology, Tehran, Iran, in 1991 and 1998, respectively, all in electrical engineering. Since 1990, he has been involved in several research and engineering projects at the Iran Telecommunications Research Center (ITRC). He is currently an Assistant Professor at Shahid Beheshti University, Tehran, Iran. His main areas of interest are the nonlinear simulation of microwave circuits, computational electromagnetics, and low phase noise oscillators. 